\title{
Hyperbaric oxygen relieves neuropathic pain through AKT/TSC2/mTOR pathway activity to induce autophagy
}

This article was published in the following Dove Medical Press journal: Journal of Pain Research

\author{
Yong-Da Liu' \\ Zhi-Bin Wang \\ Guang Han' \\ Li Jin ${ }^{2}$ \\ Ping Zhao'
}

'Department of Anesthesiology and Pain Management, Shengjing Hospital of China Medical University, Shenyang I l 0004, China; ${ }^{2}$ Department of Orthopaedic Surgery, University of Virginia, Charlottesville, VA 22908, USA
Correspondence: Ping Zhao

Department of Anesthesiology and Pain Management, Shengjing Hospital of China Medical University, No.36 Sanhao Street, Heping District, Shenyang I 10004, China Tel +86 I89 4025 897।

Email zhaop@sj-hospital.org
Background: Our previous study suggested that HBO treatment attenuated neuropathic pain by inhibiting mTOR to induce autophagy in SNL neuropathic pain model. The aim of this study was to evaluate the role of AKT/TSC2/mTOR pathway in SNL and autophagy and determine whether HBO treatment could relieve neuropathic pain via modulating AKT/TSC2/mTOR pathway.

Materials and methods: Rats were randomly divided into sham, SNL, SNL + HBO treatment, $\mathrm{SNL}+$ vehicle, and SNL + AKT inhibitor groups. Neuropathic pain was induced following SNL procedure. Rats in the SNL $+\mathrm{HBO}$ group received $\mathrm{HBO}$ treatment for 7 consecutive days beginning on postoperative day 1 . The SNL + vehicle group received $10 \mu \mathrm{L}$ of $3 \%$ dimethyl sulfoxide in saline. SNL + AKT inhibitor group received $10 \mu \mathrm{L}$ AKT inhibitor IV intrathecally. Mechanical withdrawal threshold tests were performed to evaluate mechanical hypersensitivity. AKT, p-AKT, TSC2, mTOR, p-mTOR, and LC3-II protein expressions were examined by Western blot analysis.

Results: HBO reversed AKT/TSC2/mTOR upregulation induced by SNL and attenuated neuropathic pain. Intrathecal injection of AKT inhibitor IV decreased the activity of AKT/TSC2/ mTOR pathway and increased LC3-II expression accompanied by analgesic effect in SNL rats. Conclusion: Taken together, our findings demonstrated AKT/TSC2/mTOR pathway was activated in SNL-induced neuropathic pain, and HBO treatment attenuated neuropathic pain via neutralizing AKT/TSC2/mTOR pathway activation.

Keywords: neuropathic pain, autophagy, mTOR, TSC2, AKT, hyperbaric oxygen

\section{Introduction}

Autophagy is one of the major mechanisms for degradation and recycling of cellular constituents, which ensure cellular differentiation, homeostasis, and survival. Autophagy dysregulation has been found in a number of pathological conditions. Numerous studies indicated that autophagy in the spinal cord was impaired in neuropathic pain models, and raising the level of autophagy in the spinal cord and dorsal root ganglion (DRG) in neuropathic pain models could alleviate hyperalgesia and allodynia. ${ }^{1,2}$ Evidence showed that autophagy might be a protective reaction in neuropathic pain. ${ }^{3,4}$

AKT pathway plays important roles in many cellular processes, including autophagy. AKT (protein kinase B/AKT) is a serine-threonine kinase, the activity of which is modulated by PI3K. ${ }^{5,6} \mathrm{PI} 3 \mathrm{~K}$ acts as an essential link between AKT and mTOR through inactivation of the TSC2. ${ }^{7}$ TSC2 inactivation may reduce its GAP activity toward Rheb and lead to the activation of mTOR.$^{8-10}$ mTOR induces the phosphorylation of its effector proteins, which subsequently inhibits ULK1/2 complex, a complex needed 
in the early steps of autophagy. ${ }^{11,12}$ In short, downregulation of AKT/TSC2/mTOR activity induces autophagy.

Emerging evidence suggest that AKT pathway plays an essential role in neuronal circuits of pain processing in the spinal cord and dorsal horn. ${ }^{13,14}$ The activation of AKT leads to chronic pain processing. ${ }^{15-17}$ Intrathecal administration of AKT inhibitors attenuated nerve injury-triggered neuropathic pain. ${ }^{18}$ Moreover, studies suggested that some medications attenuated neuropathic pain by inhibiting AKT signaling pathway. For example, Hourruynia cordata Thunb. could reverse the hypersensitivity induced by oxaliplatin, an antiadvanced colorectal cancer drug, via downregulating PI3K/ AKT/mTOR signaling pathway. ${ }^{19}$ Also, celecoxib alleviated neuropathic pain in the DRG of oxaliplatin-induced neuropathic pain model by inhibiting AKT signaling. ${ }^{14}$ Atorvastatin prevented neuroinflammation via attenuating $\mathrm{p}-\mathrm{AKT} / \mathrm{AKT}$ upregulation in CCI neuropathic pain model. ${ }^{15}$ In addition, SDF-1-CXCR4 signaling mediated the transition from acute to chronic pain state and contributed to the onset and maintenance of neuropathic pain via modulating AKT signaling pathway. $^{20}$

HBO is a noninvasive treatment which provides $100 \%$ oxygen at a greater pressure than that at the sea level, usually 2.0-2.5 ATA, in animal experiments. It has been suggested that $\mathrm{HBO}$ treatment can reduce pain in animal models and modulate pain in humans as well. ${ }^{21} \mathrm{HBO}$ treatment inhibited astrocyte profiling and inflammation and influenced iNOS and nNOS expressions. ${ }^{22,23}$ Furthermore, a study outlined that NO-cGMP-PKG signal transduction pathway was involved in HBO treatment. ${ }^{24}$ Of note, our team demonstrated that HBO therapy palliated CCI-induced neuropathic pain in rats by upregulating microglial mitophagy. ${ }^{25}$ Furthermore, our previous work showed that the level of spinal cord autophagy could be elevated by HBO treatment to produce antinociceptive effects via inhibiting mTOR pathway in neurons. ${ }^{26}$ The aim of this study was to investigate the role of AKT/TSC $2 / \mathrm{mTOR}$ pathway in SNL-induced neuropathic pain and whether $\mathrm{HBO}$ treatment could induce autophagy to attenuate neuropathic pain via AKT/TSC2/mTOR pathway.

In this study, we found that the phosphorylation level of $\mathrm{AKT} / \mathrm{TSC} 2 / \mathrm{mTOR}$ was significantly increased in the spinal cord of SNL group accompanied by deceased MWT in the hind paw. Intrathecal injection of AKT inhibitor IV activated autophagy in the spinal cord via inhibiting AKT/ TSC2/mTOR pathway. Moreover, HBO treatment attenuated the mechanical hypersensitivity and increased spinal autophagy by neutralizing SNL-induced activity of AKT/ TSC2/mTOR pathway.

\section{Materials and methods}

\section{Animals}

This study was carried out in strict accordance with the recommendations in the guide for the care and use of laboratory animals of China Medical University. The protocol was approved by the Institutional Animal Ethics Committee of China Medical University (approval number: 2016PS013K). The surgery was performed under $1 \%$ pentobarbital sodium anesthesia (35 mg/kg intraperitoneally). The procedure was designed to minimize animal's suffering. Adult male Sprague Dawley rats (200-220 g; Changsheng Bio-Technology Co Ltd, Changchun, China) were housed individually in a humidity-controlled room at $23^{\circ} \mathrm{C}-25^{\circ} \mathrm{C}$ with a 12 -hour light/ dark cycle. Animals had free access to food and water ad libitum. All rats were randomly assigned to different groups.

\section{SNL model}

According to the procedure originally proposed by Kim and Chung, the left fifth lumbar spinal nerve was tightly ligated by a 4-0 silk suture and cut distal to the ligation. ${ }^{27}$ The surgical procedure performed in the sham group was identical to the SNL group, except that the spinal nerve was not ligated.

\section{Behavioral assessment of mechanical sensitivity}

Animals were placed in Plexiglas chambers on an elevated wire grid and allowed to habituate for at least 30 minutes. MWT was defined as the minimum force $(\mathrm{g})$ with which a von Frey filament (0.4-15 g; Stoelting Company, Wood Dale, IL, USA) triggered a positive paw withdrawal response according to an up-and-down procedure. ${ }^{28} \mathrm{~A}$ positive response was defined as a quick withdrawal of the hind paw upon stimulation. The behavioral tests were carried out before SNL surgery and at 1, 3, 5, and 7 days after SNL.

\section{HBO treatment}

The HBO treatment chamber (DS400-IV; Weifang Huaxin Oxygen Industry Co. Ltd., Shandong, China) was precoated with soda lime flushed with pure oxygen for 15 minutes. After the rats were placed in the chamber, the pressure in the chamber was increased to 2.0 ATA in 15 minutes and maintained for 60 minutes. The chamber was then decompressed to normal room pressure in 15 minutes. SNL + HBO group received HBO treatment once a day for 7 consecutive days from postoperative day 1. Rats in the sham, SNL, SNL + vehicle, and $\mathrm{SNL}+\mathrm{AKT}$ inhibitor groups were placed inside the chamber for 90 minutes without $\mathrm{HBO}$ treatment as controls. 


\section{Intrathecal drug delivery}

Intrathecal injection was performed as described previously. ${ }^{19}$ Briefly, under anesthesia, laminectomy was performed at the L5 vertebra. PE-5 catheter was inserted into the subarachnoid space of the spinal cord at L4 level. The AKT inhibitor IV $(0.8 \mu \mathrm{g} / 10 \mu \mathrm{L}$; Sigma-Aldrich) was administered on postoperative days 1,3 , and 5 in SNL + AKT inhibitor group. The SNL + vehicle group received $10 \mu \mathrm{L}$ of $3 \%$ dimethyl sulfoxide in saline.

\section{Western blot analysis}

At the harvest time points, rats were sacrificed and the spinal cord lumbar segment (L4-L5) was carefully dissected and immediately frozen at $-80^{\circ} \mathrm{C}$ until use. The specimens were homogenized in ice-cold RIPA lysis buffer (p0013B; Beyotime, Beijing, China) in the presence of protease inhibitors (cod. P8349; Sigma-Aldrich) and incubated on ice for 30 minutes followed by centrifugation at $14,000 \mathrm{rpm}$ for 30 minutes at $4^{\circ} \mathrm{C}$. After centrifugation, the supernatants were collected. Equal amounts of total protein were separated by $10 \%$ SDS-PAGE and transferred onto polyvinylidene difluoride membrane (Immobilon-P; EMD Millipore, Billerica, MA, USA). The membrane was incubated with $5 \%$ nonfat milk in Tris-buffered saline containing $0.05 \%$ Tween 20 to block nonspecific signals. The membrane was then incubated with following primary antibodies overnight at $4{ }^{\circ} \mathrm{C}$ : rabbit anti-AKT (1:1,000, 9272s; Cell Signaling Technology); rabbit anti-p-AKT (1:1,000, 4060s; Cell Signaling Technology); rabbit anti-LC3 (1:1,000, 2775s; Cell Signaling Technology); rabbit anti-mTOR $(1: 1,000,2983 \mathrm{~s}$; Cell Signaling Technology); rabbit anti-p-mTOR $(1: 1,000,2971$ s; Cell Signaling Technology); rabbit anti-TCS (1:1,000, 4308s; Cell Signaling Technology); and mouse anti-GAPDH (1:10,000; Vazyme, Nanjing, China). The membranes were incubated with corresponding horse radish peroxidase-conjugated secondary antibody for 1 hour at room temperature (anti-rabbit 1:2,000 [ZB-2301] and anti-mouse 1:2,000 [ZB-2305]; ZSGB-BIO, Beijing, China). The protein signal was visualized using the ECL Western Blotting Detection kit (NC15079; Thermo Fisher Scientific, Waltham, MA, USA). Images were acquired with imaging system (c300; Azure Biosystems, Dublin, CA, USA) and quantified with ImageJ software (NIH, Bethesda, MD, USA).

\section{Statistical analysis}

Statistical analysis was performed with IBM SPSS Statistics 22 software. Data were analyzed using paired Student's $t$-test for two groups and one-way ANOVA for more than two groups. The results were expressed as the mean \pm SEM. $P$-values $<0.05$ were considered significant.

\section{Results \\ SNL induced a severe mechanical hyperalgesia}

To verify the development of neuropathic pain after nerve ligation, we tested the mechanical sensitivity in animals with von Frey filaments. As reported previously, we confirmed that nerve ligation to the spinal nerve caused significant decreases in paw withdrawal threshold in the left paw (SNL) as compared to the left paw of the sham group (sham) on day 1, and the withdrawal threshold progressively decreased up to day 7 (Figure 1A, $\mathrm{n}=5, P<0.05$ ). The withdrawal threshold of both paws of sham group (sham and sham-R) and contralateral paws of SNL group (SNL-R) remained unchanged (Figure $1 \mathrm{~A}, \mathrm{n}=5, P>0.05)$.

\section{HBO reversed AKT/TSC2/mTOR upregulation induced by SNL}

Compared to the sham group, our data demonstrated the levels of p-AKT/AKT (Figure 2B, 1.36 $\pm 0.32, \mathrm{n}=7, P<0.05$ ) and $\mathrm{p}-\mathrm{mTOR} / \mathrm{mTOR}$ (Figure 2D, $1.75 \pm 0.38, \mathrm{n}=6, P<0.05$ ) were upregulated in the spinal cord of SNL group. In addition, our finding indicated that TSC2 was downregulated in the SNL group than sham group (Figure $2 \mathrm{C}, 0.66 \pm 0.19, \mathrm{n}=6, P<0.05$ ) on postoperative day 7. However, HBO treatment reversed these effects induced by SNL. Compared with SNL group, the phosphorylation level of AKT/TSC2/mTOR was significantly decreased in SNL + HBO group (Figure 2, 0.68 \pm 0.14 , $1.80 \pm 0.68,0.64 \pm 0.20, \mathrm{n}=6, P<0.05)$. As expected, SNL + HBO significantly increased paw withdrawal threshold in comparison to SNL group. The difference was observed after HBO treatment and persistent throughout the observation period till day 7 (Figure $1 \mathrm{~B}, 0.55 \pm 0.11, \mathrm{n}=6, P<0.05$ ). MWT recordings were stable at the baseline in paws among sham, $\mathrm{SNL}$, and $\mathrm{SNL}+\mathrm{HBO}$ groups (Figure $1 \mathrm{~B}, \mathrm{n}=6, P<0.05$ ).

\section{AKT inhibitor alleviated mechanical hypersensitivity induced by SNL via inhibiting AKT/TSC2/mTOR}

Studies indicated that AKT pathway was upregulated in SNLinduced neuropathic pain rats. ${ }^{29}$ To further determine whether AKT/TSC2/mTOR pathway contributes to the mechanical hypersensitivity induced by SNL, we examined protein expressions following the administration of AKT inhibitor. In this study, the AKT inhibitor IV was given intrathecally on postop- 


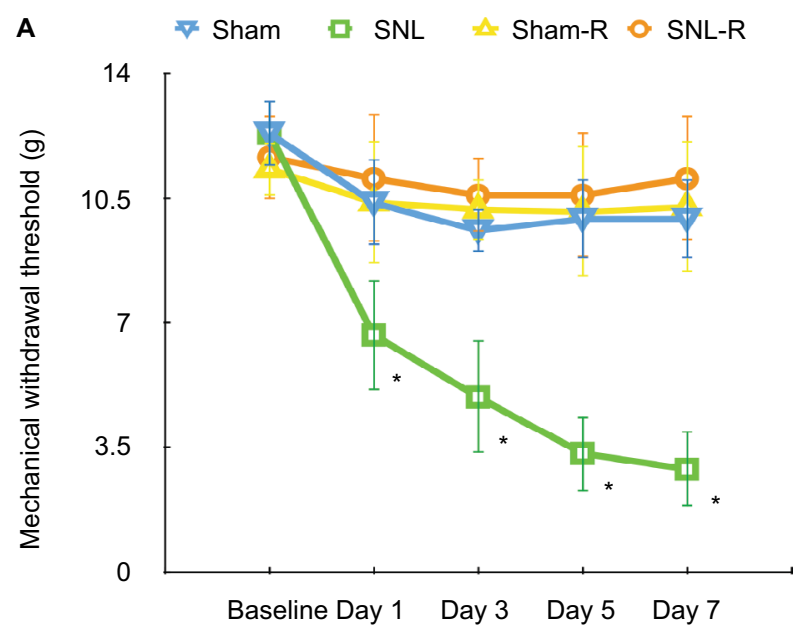

B

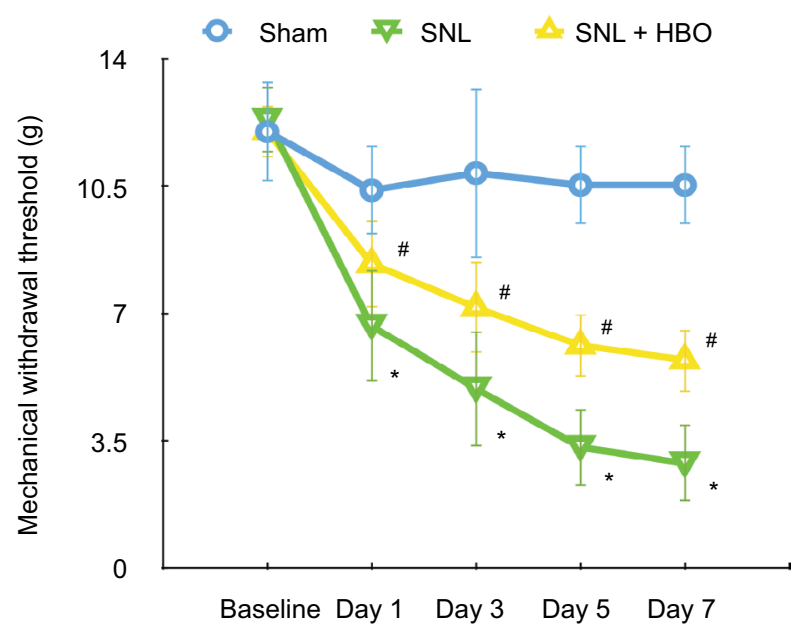

C

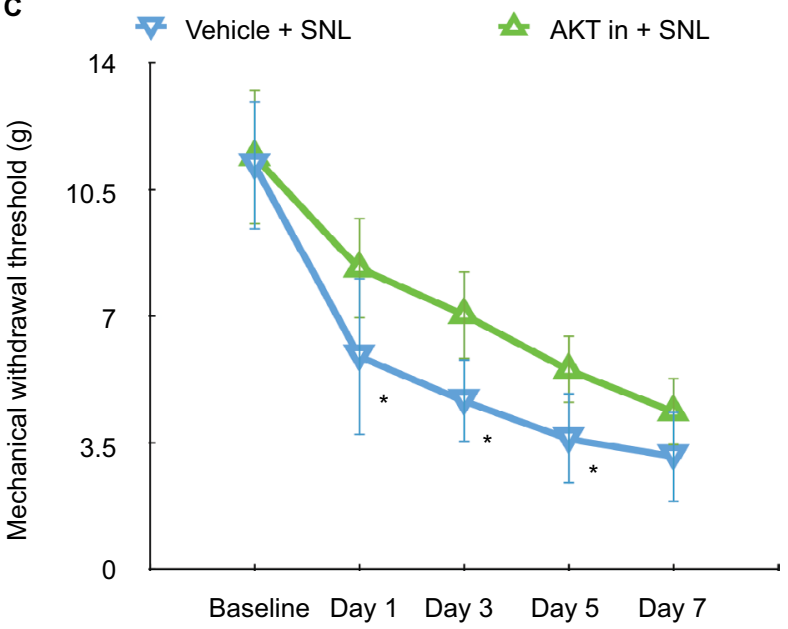

Figure I (A) MWT in sham (ipsilateral side of sham group), sham-R (contralateral side of sham group), SNL (ipsilateral side of SNL group), and SNL-R (contralateral side of SNL group) groups. $n=5$ per group. ${ }^{*} P<0.05$ vs sham. The MWT of both paws of sham group and contralateral paws of SNL group remained unchanged. (B) The effects of HBO treatment following SNL on MWT in rats in sham, SNL, and SNL + HBO groups. $n=5$ per group. ${ }^{*} P<0.05$ vs sham, ${ }^{\#}<<0.05$ vs $S N L+H B O$ group. (C) The effects of intrathecal injection of AKT inhibitor IV on the neuropathic painrelated behaviors induced by $S N L$. $n=5$ per group. $* P<0.05$ vs vehicle injection group. erative days 1, 3, and 5. As shown in Figure 1C, the withdrawal threshold was significantly increased at inhibitor administration on day 1, and this effect maintained till day 5 (Figure 1C, $1.58 \pm 0.38, \mathrm{n}=7, P<0.05)$. As shown in Figure 3, the p-AKT (Figure 3, $0.68 \pm 0.14, \mathrm{n}=5, P<0.05$ ) and $\mathrm{p}$-mTOR (Figure 3, $0.64 \pm 0.08, \mathrm{n}=5, P<0.05)$ were significantly decreased in the inhibitor group compared to the vehicle group. In contrast, the protein expression of TSC 2 (Figure $3,1.80 \pm 0.68, \mathrm{n}=5, P<0.05$ ) was significantly increased after inhibitor administration. All these data suggested that upregulation of AKT/TSC2/mTOR led to neuropathic pain aggravation.

\section{Intrathecal injection of AKT inhibitor IV induced autophagy}

In addition to the changes in AKT activity and mechanical sensitivity, application of AKT inhibitor IV also induced autophagy of the spinal cord. As shown in Figure 3, LC3-II was significantly induced after treatment with AKT inhibitor. (Figure 3, 1.45 $\pm 0.29, \mathrm{n}=5, P<0.05$ ). These data suggest that autophagy was inversely associated with the AKT/TSC2/ mTOR pathway in this neuropathic pain model (Figure 3, $0.57 \pm 0.16,1.62 \pm 0.37,0.71 \pm 0.20, \mathrm{n}=5, P<0.05$ ).

\section{Discussion}

Neuropathic pain is a chronic state caused by nervous system lesions. Current treatments of neuropathic pain focus on relieving pain. Multimodal pharmacological treatments for neuropathic pain involve the use of antiepileptics, antidepressants, opioid analgesics, and local anesthetics. ${ }^{14}$ However, drug therapies have limited efficacy and doselimiting toxic effects. ${ }^{30}$ Plasticity of the CNS may become detrimental to the nervous system, causing significant dysfunctions. In this case, neuropathic pain is the result of maladaptive plasticity leading to peripheral and central sensitization. Patients suffer from varying neuropathic pain syndromes, resulting in an enormous medical cost and psychological burden. This highlights the need to find novel interventions and treatments to reduce the burden of neuropathic pain. ${ }^{31}$ The complex underlying mechanisms are far from clear. In recent years, the role of autophagy in neuropathic pain has received attention because of its potential clinical value. Autophagy is an evolutionarily conserved and cellular self-cannibalization process that acts as an indispensable mechanism to maintain cellular homeostasis by promoting degradation pathway of autolysosomes and recycling of catabolized intracellular components. ${ }^{17,32}$ Autophagy responds to different regulatory factors such as 


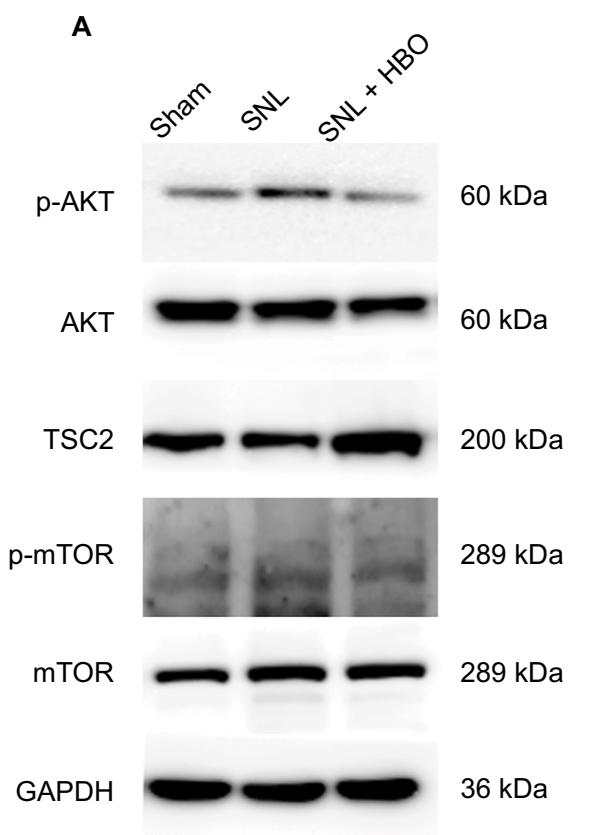

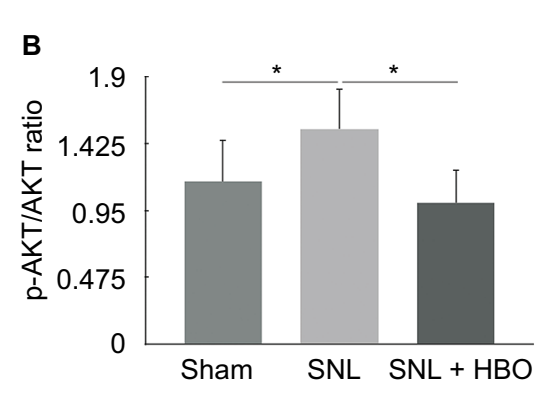

C
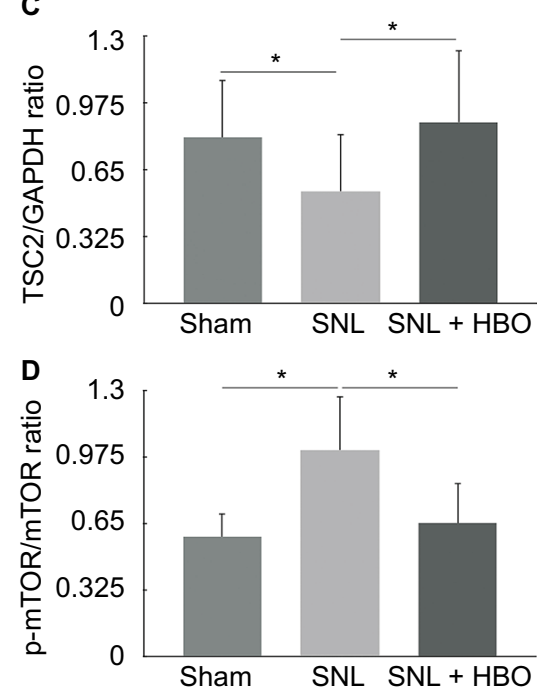

Figure 2 (A) Western Blot quantification of p-AKT, AKT, TSC2, p-mTOR, and mTOR in sham, SNL, and SNL + HBO groups. GAPDH was used as a sample loading control. (B) $\mathrm{p}$-AKT/AKT ratio. (C) TSC2/GAPDH ratio. (D) $\mathrm{p}-\mathrm{mTOR} / \mathrm{mTOR}$ ratio. ${ }^{*} \mathrm{p}<0.05$ was considered significant. $\mathrm{n}=6$ per group.

AKT pathway. AKT is an important major negative regulator of TSC2. Downregulation of TSC2 may reduce its GAP activity toward Rheb, subsequently leading to activation of mTOR, which leads to the inactivation of ULK1, thus decreasing the level of autophagy. ${ }^{33,34}$

Autophagy plays important roles in many physiological and pathological processes. Of note, a study demonstrated that autophagy is dysfunctional and impaired in neuropathic pain. ${ }^{35}$ Autophagy was found to primarily act as a protective mechanism that could relieve hyperalgesia and allodynia induced by neuropathic pain. ${ }^{2}$ LC3-II and p62 were upregulated in an SNL animal model, suggesting that autophagy was defective in autophagosomes clearance in the neuropathic pain. ${ }^{36}$ In this study, our data confirmed that autophagy was impaired in SNL neuropathic pain model. Moreover, studies uncovered that autophagy process was disrupted in neuropathic pain, localized on neurons, GABAergic interneurons, astrocytes, microglia, and Schwann cells. ${ }^{2,37,38}$ Studies also indicated that treatments increased autophagy activity, prevented the onset and delayed the chronification of neuropathic pain, and ameliorated neuropathic pain-related behaviors. ${ }^{3,39}$
HBO was developed as a treatment of decompression sickness, but it is also being recognized as a noninvasive modality for chronic pain. ${ }^{22}$ Current evidence indicates that HBO provides long-lasting efficacy and has minimal side effects in treating neuropathic pain from lab bench to bedside. ${ }^{22}$ Studies uncovered that HBO relieves neuropathic pain via glial cells and vascular system-related proteins and signaling pathway. Zhao et al demonstrated that HBO reversed inflammatory signaling and astrocyte activation. ${ }^{40,41}$ Ding et al indicated that $\mathrm{HBO}$ alleviated neuropathic pain via decreasing expressions of iNOS and nNOS and reducing levels of inflammatory factors. ${ }^{23,29}$ Lim et al suggested that HBO reversed vascular system dysfunction and endoneurial hypoxia in neuropathic pain model. ${ }^{42}$ Recently, our team outlined that $\mathrm{HBO}$ elevated the level of mitophagy, a type of selective autophagy, and palliated CCI-induced neuropathic pain. ${ }^{25}$ Furthermore, our previous work indicated that HBO relieved neuropathic pain by elevating autophagy flux via inhibiting mTOR and downstream pathway in neurons. ${ }^{26}$ Blockage of autophagy activity with chloroquine partly neutralized the treatment effect of $\mathrm{HBO}$, while rapamycin enhanced the analgesic effect of $\mathrm{HBO} .{ }^{26}$ To further determine 
A

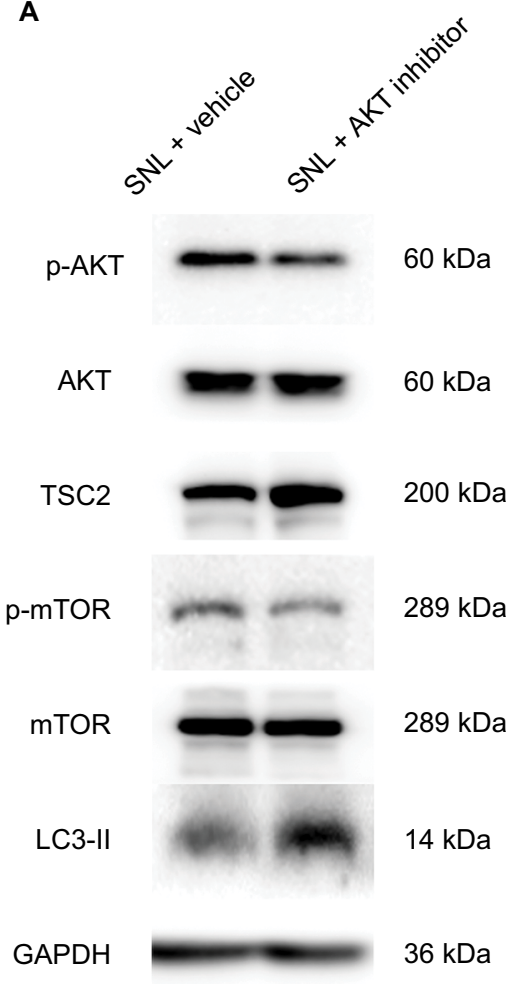

B

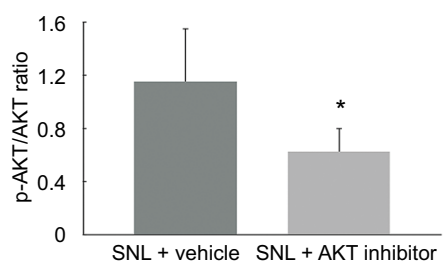

C

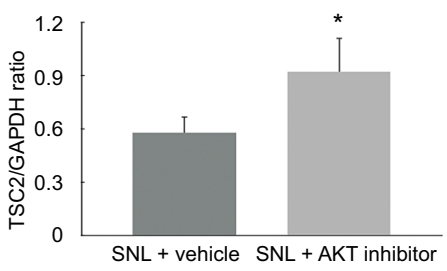

D

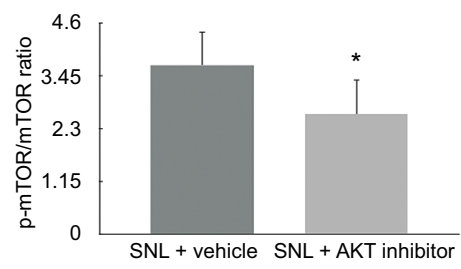

E

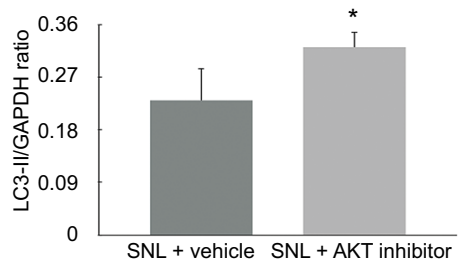

Figure 3 (A) The expression of AKT/TSC2/mTOR pathway was analyzed in vehicle + SNL group and AKT inhibitor + SNL group. (B) p-AKT/AKT ratio was decreased by AKT inhibitor. (C) TSC2/GAPDH ratio was increased significantly. (D) p-mTOR/mTOR ratio was decreased. (E) LC3-II/GAPDH ratio was used to evaluate the spinal cord autophagy. Data showed that AKT inhibitor induced autophagy by decreasing AKT/TSC2/mTOR pathway activity in SNL spinal cord. $* P<0.05$ was considered significant. $\mathrm{n}=5$ per group.

whether AKT/TSC2/mTOR regulates the analgesic effect of $\mathrm{HBO}$ in neuropathic pain, we conducted further research to extend our understanding of how HBO elevates spinal cord autophagy flux. Our data showed that SNL induced AKT/ TSC2/mTOR pathway activation compared to sham group, the phosphorylation level of AKT/TSC2/mTOR was significantly decreased in SNL + HBO group, and neuropathic pain-related behavior tests were ameliorated in comparison to SNL group. Our observations suggested that $\mathrm{HBO}$ provided analgesic effect via reversing AKT/TSC2/mTOR upregulation induced by SNL. This study is the first to analyze how HBO modulates AKT/TSC2/mTOR pathway activity in neuropathic pain model rats.

To further understand the role of AKT/TSC2/mTOR pathway in neuropathic pain, we blocked AKT activity with AKT inhibitor IV in SNL rats. The phosphorylation level of mTOR was decreased in AKT inhibitor-treated SNL animals compared to the control group, while the expres- sion level of TSC2 was increased in AKT inhibitor-treated SNL group. These data suggested that the expression level of TSC2 was inversely correlated with p-AKT in the SNL model. We further analyzed the autophagy activity in AKT inhibitor-treated SNL model by measuring LC3-II level by Western blotting. Consistent with other studies, the level of LC3-II, the autophagosome-associated LC3 form, was increased in AKT inhibitor-treated SNL group in comparison to the vehicle control group, which indicates AKT inhibitor could induce autophagy. Furthermore, the behavioral tests showed that AKT inhibitor increased MWT at day 1 which was maintained till day 5 after SNL. Our study shows for the first time that the level of AKT and mTOR is increased and TSC2 is downregulated in SNL group compared to the sham group. In short, intrathecal administration of AKT inhibitor IV alleviated mechanical hypersensitivity induced by SNL and increased LC3-II expression, suggesting that inhibition of AKT/TSC2/ 
mTOR pathway might be a prominent autophagic pathway to alleviate neuropathic pain.

There is no doubt that clinicians only receive partially effective outcome by traditional pharmacological treatments for neuropathic pain syndromes. We suggest that the future investigations should focus on specific cellular targets underlying chronic pain, including neuropathic pain. mTOR is now officially known as "mechanistic" target of rapamycin. ${ }^{34}$ $\mathrm{mTOR}$ and related pathway could be essential as the regulator of protein synthesis, involved in the process of neuronal functions, such as synaptic plasticity in the CNS and peripheral nervous system, which is a major mechanism of onset, development, and maintenance of neuropathic pain. ${ }^{30} \mathrm{mTOR}$ and its downstream pathways were activated in inflammatory pain and neuropathic pain. ${ }^{26,43}$ Studies show that mTOR inhibitors are promising novel pharmacotherapies for pain management, and are also approved for clinical use. ${ }^{29,43-45}$ However, in this study, we focused on the upstream pathway of mTOR and HBO treatment.

As mentioned above, the upstream pathway of mTOR, AKT-related pathway, plays an important role in treatment effect as well. Of note, neuropathic pain might be secondary to TSC, an autosomal dominant disorder. Rapamycin is identified as an effective and safe treatment in clinic. ${ }^{44,45} \mathrm{AKT} /$ TSC2/mTOR might be a promising target signaling pathway and could provide a precision treatment for TSC-induced neuropathic pain. To sum up, AKT/TSC2/mTOR might be a potential treatment target for neuropathic pain.

However, AKT is not the only pathway to increase mTOR activity via promoting the degradation of the TSC $1 / 2$ complex. AMPK $\alpha$ (also known as PRKAA) and ERK1 and 2 can modulate the phosphorylation and degradation of $\mathrm{mTOR}$ as well. ${ }^{46,47}$ The pathway network involving them still remains unknown and warrants further investigations. Whether other physiological process, like endoplasmic reticulum stress and apoptosis, could modulate and/or interact with autophagy via mTOR pathway in neuropathic pain needs to be further elucidated. On the other hand, AKT inhibitor IV provided treatment effect from day 1 to day 5, while HBO relieved mechanical allodynia from day 1 to day 7 . Further studies on the effect of different pharmacotherapies and the effect of HBO are needed as well.

There is no doubt that LC3-II is a key protein to evaluate the function of autophagy. In this study, we demonstrated the administration of AKT inhibitor IV increased LC3-II using Western blot analysis. Data suggested that autophagosomes formation was elevated, but just examined autophagosomes level might not evaluate the whole process of autophagy. Further studies on the stages of autophagic process are required to confirm the findings of our study.

Health care providers treating neuropathic pain might not receive a satisfied response from patients following traditional clinical treatments. Novel therapies such as rapamycin and stem cell injection might be promising for pain management. ${ }^{48}$ In addition to $\mathrm{HBO}$, we will also discuss the underlying mechanisms of other clinical treatments in the near future.

\section{Conclusion}

This study demonstrated that AKT/TSC2/mTOR pathway was upregulated in SNL-induced neuropathic pain model. AKT inhibitor relieved neuropathic pain by inhibiting AKT/ TSC2/mTOR to induce autophagy. Furthermore, HBO alleviated neuropathic pain-related behavior via autophagic pathway. This study is the first to analyze AKT/TSC2/ mTOR in neuropathic pain and HBO treatment, and extends our understanding of how HBO modulates autophagy in neuropathic pain.

\section{Abbreviations}

$\mathrm{CCI}$, chronic constriction injury; CNS, central nervous system; HBO, hyperbaric oxygen; MWT, mechanical withdrawal threshold; SNL, spinal nerve ligation; TSC, tuberous sclerosis complex

\section{Data sharing statement}

All relevant data are within the paper.

\section{Acknowledgments}

The authors thank Professor Lili Wang, Xingyue Li, Jiao Guo, Yi Cici Zhang, and Jianing Miao for the support. Yong-Da Liu wishes to thank Shuang Wang for support. This research is supported by grants from the National Natural Science Foundation of China (No.81671311; No.81600971) and Outstanding Scientific Fund of Shengjing Hospital (No. 201708).

\section{Author contributions}

All authors contributed to data analysis, drafting and revising the article, gave final approval of the version to be published, and agree to be accountable for all aspects of the work.

\section{Disclosure}

The authors report no conflicts of interest in this work. 


\section{References}

1. Berliocchi L, Maiarù M, Varano GP, et al. Spinal autophagy is differently modulated in distinct mouse models of neuropathic pain. Mol Pain. 2015;11:1744-8069.

2. Marinelli S, Nazio F, Tinari A, et al. Schwann cell autophagy counteracts the onset and chronification of neuropathic pain. Pain. 2014;155(1):93-107.

3. Guo JS, Jing PB, Wang JA, et al. Increased autophagic activity in dorsal root ganglion attenuates neuropathic pain following peripheral nerve injury. Neurosci Lett. 2015;599:158-163.

4. Jung KT, Lim KJ. Autophagy: can it be a new experimental research method of neuropathic pain? Korean J Pain. 2015;28(4):229-230.

5. Xu B, Guan XH, Yu JX, et al. Activation of spinal phosphatidylinositol 3-kinase/protein kinase B mediates pain behavior induced by plantar incision in mice. Exp Neurol. 2014;255:71-82.

6. Yu LN, Zhou XL, Yu J, et al. PI3K contributed to modulation of spinal nociceptive information related to ephrinBs/EphBs. PLoS One. 2012;7(8):e40930.

7. Song MS, Salmena L, Pandolfi PP. The functions and regulation of the PTEN tumour suppressor. Nat Rev Mol Cell Biol. 2012;13(5):283-296.

8. Parkhitko A, Myachina F, Morrison TA, et al. Tumorigenesis in tuberous sclerosis complex is autophagy and p62/sequestosome 1 (SQSTM1)dependent. Proc Natl Acad Sci U SA. 2011;108(30):12455-12460.

9. Cabrera-López C, Bullich G, Martí T, et al. Insight into response to mTOR inhibition when PKD1 and TSC2 are mutated. BMC Med Genet. 2015;16(1):39.

10. Dimario FJ, Sahin M, Ebrahimi-Fakhari D. Tuberous sclerosis complex. Pediatr Clin North Am. 2015;62(3):633-648.

11. Mao XY, Zhou HH, Li X, Liu ZQ. Huperzine a alleviates oxidative glutamate toxicity in hippocampal HT22 cells via activating BDNF/ TrkB-dependent PI3K/Akt/mTOR signaling pathway. Cell $\mathrm{Mol} \mathrm{Neu-}$ robiol. 2016;36(6):915-925.

12. Huang H, Kang R, Wang J, Luo G, Yang W, Zhao Z. Hepatitis C virus inhibits AKT-tuberous sclerosis complex (TSC), the mechanistic target of rapamycin (MTOR) pathway, through endoplasmic reticulum stress to induce autophagy. Autophagy. 2013;9(2):175-195.

13. Shi TJ, Huang P, Mulder J, Ceccatelli S, Hökfelt T. Expression of p-Akt in sensory neurons and spinal cord after peripheral nerve injury. Neurosignals. 2009;17(3):203-212.

14. Jiang SP, Zhang ZD, Kang LM, Wang QH, Zhang L, Chen HP. Celecoxib reverts oxaliplatin-induced neuropathic pain through inhibiting PI3K/ Akt2 pathway in the mouse dorsal root ganglion. Exp Neurol. 2016;275 (Pt 1):11-16.

15. Chu LW, Chen JY, Wu PC, Wu BN, Pc W, Bn W. Atorvastatin prevents neuroinflammation in chronic constriction injury rats through nuclear $\mathrm{NF} \kappa \mathrm{B}$ downregulation in the dorsal root ganglion and spinal cord. $A C S$ Chem Neurosci. 2015;6(6):889-898.

16. Popiolek-Barczyk K, Mika J. Targeting the microglial signaling pathways: new insights in the modulation of neuropathic pain. Curr Med Chem. 2016;23(26):2908-2928.

17. Mh K, Yang ML, Youn SC, Lan CT, Tseng TJ. Intact subepidermal nerve fibers mediate mechanical hypersensitivity via the activation of protein kinase C gamma in spared nerve injury. Mol Pain. 2016;12.

18. Xu JT, Tu HY, Xin WJ, Liu XG, Zhang GH, Zhai CH. Activation of phosphatidylinositol 3-kinase and protein kinase B/Akt in dorsal root ganglia and spinal cord contributes to the neuropathic pain induced by spinal nerve ligation in rats. Exp Neurol. 2007;206(2):269-279.

19. Wan CF, Zheng LL, Liu Y, Yu X. Houttuynia cordata Thunb reverses oxaliplatin-induced neuropathic pain in rat by regulating Th17/Treg balance. Am J Transl Res. 2016;8(3):1609-1614.

20. Yang F, Sun W, Luo WJ. SDF1-CXCR4 signaling contributes to the transition from acute to chronic pain state. Mol Neurobiol. 2016.

21. Sutherland AM, Clarke HA, Katz J, Katznelson R. Hyperbaric oxygen therapy: a new treatment for chronic pain? Pain Pract. 2016;16(5): $620-628$.
22. Zhao BS, Meng LX, Ding YY, Cao YY. Hyperbaric oxygen treatment produces an antinociceptive response phase and inhibits astrocyte activation and inflammatory response in a rat model of neuropathic pain. J Mol Neurosci. 2014;53(2):251-261.

23. Ding Y, Yao P, Hong T, et al. The analgesic effect of early hyperbaric oxygen treatment in chronic constriction injury rats and its influence on nNOS and iNOS expression and inflammatory factor production. Mol Pain. 2018;14:174480691876583.

24. Ding Y, Yao P, Hong T, Han Z, Zhao B, Chen W. The NO-cGMP-PKG signal transduction pathway is involved in the analgesic effect of early hyperbaric oxygen treatment of neuropathic pain. J Headache Pain. 2017;18(1):51.

25. Han G, Liu K, Li L, Li X, Zhao P. Effects of hyperbaric oxygen therapy on neuropathic pain via mitophagy in microglia. Molecular Pain. 2017;13:174480691771086.

26. Liu YD, Wang ZB, Han G, Zhao P. Hyperbaric oxygen treatment attenuates neuropathic pain by elevating autophagy flux via inhibiting mTOR pathway. Am J Transl Res. 2017;9(5):2629-2638.

27. Kim SH, Chung JM. An experimental model for peripheral neuropathy produced by segmental spinal nerve ligation in the rat. Pain. 1992;50(3):355-363.

28. Chaplan SR, Bach FW, Pogrel JW, Chung JM, Yaksh TL. Quantitative assessment of tactile allodynia in the rat paw. J Neurosci Methods. 1994;53(1):55-63.

29. Kondo D, Saegusa H, Tanabe T. Involvement of phosphatidylinositol-3 kinase/Akt/mammalian target of rapamycin/peroxisome proliferatoractivated receptor $\gamma$ pathway for induction and maintenance of neuropathic pain. Biochem Biophys Res Commun. 2018;499(2):253-259.

30. Gilron I, Jensen TS, Dickenson AH. Combination pharmacotherapy for management of chronic pain: from bench to bedside. Lancet Neurol. 2013;12(11):1084-1095.

31. Lisi L, Aceto P, Navarra P, Dello Russo C. mTOR Kinase: a possible pharmacological target in the management of chronic pain. Biomed Res Int. 2015;2015(6):1-13.

32. Hansen M, Rubinsztein DC, Walker DW. Autophagy as a promoter of longevity: insights from model organisms. Nat Rev Mol Cell Biol. 2018.

33. Fernández ÁF, Sebti S, Wei Y, et al. Disruption of the beclin 1-BCL2 autophagy regulatory complex promotes longevity in mice. Nature. 2018;558(7708):136-140.

34. Potter LE, Paylor JW, Suh JS, et al. Altered excitatory-inhibitory balance within somatosensory cortex is associated with enhanced plasticity and pain sensitivity in a mouse model of multiple sclerosis. J Neuroinflammation. 2016;13(1):142.

35. Altmann C, Hardt S, Fischer C, et al. Progranulin overexpression in sensory neurons attenuates neuropathic pain in mice: Role of autophagy. Neurobiol Dis. 2016;96:294-311.

36. Berliocchi L, Russo R, Maiarù M, Levato A, Bagetta G, Corasaniti MT. Autophagy impairment in a mouse model of neuropathic pain. Mol Pain. 2011;7:1744-8069.

37. Zhang E, Yi MH, Ko Y, et al. Expression of LC3 and Beclin 1 in the spinal dorsal horn following spinal nerve ligation-induced neuropathic pain. Brain Research. 2013;1519:31-39.

38. Shi G, Shi J, Liu K, et al. Increased miR-195 aggravates neuropathic pain by inhibiting autophagy following peripheral nerve injury. Glia. 2013;61(4):504-512.

39. Wang H, Huo X, Chen H. Hydrogen-rich saline activated autophagy via HIF-1a pathways in neuropathic pain model. Biomed Res Int. 2018.

40. Zhao BS, Meng LX, Ding YY, Cao YY. Hyperbaric oxygen treatment produces an antinociceptive response phase and inhibits astrocyte activation and inflammatory response in a rat model of neuropathic pain. J Mol Neurosci. 2014;53(2):251-261.

41. Zhao B, Pan Y, Xu H, Song X. Hyperbaric oxygen attenuates neuropathic pain and reverses inflammatory signaling likely via the Kindlin-1/ Wnt-10a signaling pathway in the chronic pain injury model in rats. $J$ Headache Pain. 2017;18(1):1. 
42. Lim TK, Shi XQ, Johnson JM, et al. Peripheral nerve injury induces persistent vascular dysfunction and endoneurial hypoxia, contributing to the genesis of neuropathic pain. $J$ Neurosci. 2015;35(8): 3346-3359.

43. Liang L, Tao B, Fan L, Yaster M, Zhang Y, Tao Y-X. mTOR and its downstream pathway are activated in the dorsal root ganglion and spinal cord after peripheral inflammation, but not after nerve injury. Brain Research. 2013;1513:17-25.

44. Koenig MK, Bell CS, Hebert AA, et al. Efficacy and safety of topical rapamycin in patients with facial angiofibromas secondary to tuberous sclerosis complex: the TREATMENT randomized clinical trial. JAMA Dermatol. 2018;154(7):773-780.
45. Duan Z, Li J, Pang X, Wang H, Su Z. Blocking mammalian target of rapamycin (mTOR) alleviates neuropathic pain induced by chemotherapeutic bortezomib. Cell Physiol Biochem. 2018;48(1):54-62.

46. Hasanvand A, Amini-Khoei H, Hadian M-R, et al. Anti-inflammatory effect of AMPK signaling pathway in rat model of diabetic neuropathy. Inflammopharmacology. 2016;24(5):207-219.

47. Hung VK, Tai LW, Qiu Q, et al. Over-expression of astrocytic ET-1 attenuates neuropathic pain by inhibition of ERK1/2 and Akt(s) via activation of ETA receptor. Mol Cell Neurosci. 2014;60:26-35.

48. Haselbach D, Raffoul W, Larcher L, Tremp M, Kalbermatten DF, di Summa PG. Regeneration patterns influence hindlimb automutilation after sciatic nerve repair using stem cells in rats. Neurosci Lett. 2016;634:153-159.
Journal of Pain Research

\section{Publish your work in this journal}

The Journal of Pain Research is an international, peer reviewed, open access, online journal that welcomes laboratory and clinical findings in the fields of pain research and the prevention and management of pain. Original research, reviews, symposium reports, hypothesis formation and commentaries are all considered for publication

\section{Dovepress}

The manuscript management system is completely online and includes a very quick and fair peer-review system, which is all easy to use. Visit http://www.dovepress.com/testimonials.php to read real quotes from published authors. 